\title{
Cognitive Memory-inspired Sentence Ordering Model
}

\author{
Weidong Liu ${ }^{\mathrm{a}}$, Xiangfeng Luo ${ }^{\mathrm{a}, *}$, Junyu Xuan $^{\mathrm{a}}$, Zheng Xu ${ }^{\mathrm{b}}$, DanDan Jiang ${ }^{\mathrm{a}}$ \\ ${ }^{a}$ Shanghai University, Shanghai, China \\ ${ }^{b}$ The Third Research Institute of Ministry of Public Security, Shanghai, China
}

\begin{abstract}
As the novel web social media emerges on the web, large scale unordered sentences are springing up in the forms: news headlines, microblogs, comments and so on. Although these massive sentences contain rich information, their loose semantic association and highly unordered semantic organization make web users extremely difficult to capture the rich information due to the lack of semantic coherence. Sentence ordering is a significant research area focusing on obtaining coherent sentence orders which could assist web user to easily understand these unordered sentences. Although many state-of-the-art coherence-based sentence ordering approaches have been proposed, some challenging issues remain unsolved: 1) what is knowledge foundation to support semantic representation and inference in sentence ordering; 2) what are the cognitive mechanisms used to guide sentence ordering; 3) how to collaborate different cognitive mechanisms for generating a coherent sentence order. To solve these issues, we propose a cognitive memory-inspired sentence ordering model. We propose three cognitive logical structures and construct their corresponding markov random fields to support semantic representation and inference. Besides, we propose three memory-inspired modules and their corresponding learning methods. These modules collaborate with each other to generate a coherent sentence order. Comparative experiments are conducted to evaluate the effectiveness of the pro-
\end{abstract}

\footnotetext{
* Corresponding author.

Email addresses: liuwd@shu.edu.cn (Weidong Liu), luoxf@shu.edu.cn (Xiangfeng Luo), xuanjunyu@shu.edu.cn (Junyu Xuan), xuzheng@shu.edu.cn (Zheng Xu), Emily_dan@shu.edu.cn (DanDan Jiang)
}

Preprint submitted to Journal of ${ }^{A} T_{E} X$ Templates

March 15, 2016

(C) 2016. This manuscript version is made available under the Elsevier user license http://www.elsevier.com/open-access/userlicense/1.0/ 
posed model. The results show our model can obtain coherent sentence orders with higher accuracy compared with the baseline methods.

Keywords: semantic link network, semantic coherence, sentence ordering, cognitive logical structure

\section{Introduction}

With the boom of microblogs, massive unordered sentences are emerging on the web as a main message passing form. Although these sentences contain much useful information, loose semantic association and unordered sentence organi-

5 zation make web users lost in the large scale data when they face these massive unordered sentences. Web users normally expect that these sentences are well ordered according to their semantic coherence since coherent sentence orders can assist them to easily understand the content of these sentences. However, such sentence ordering problem is burdensome computation even though the sentence scale is small. For example, 15 sentences have 1,307,674,368,000 permutations and each permutation corresponds to a coherence state. Obviously, the exhaustive sentence ordering algorithm is unrealistic since it is a combinatorial explosion problem caused by two reasons:

1) little overall knowledge guides the whole sentence ordering and loose association relations between sentences hinder sentence ordering without calculating all of the possible sentence orders;

2) the cognitive mechanisms of sentence ordering are paid less attraction on and the exhaustive sentence ordering algorithm is not simplified.

The existing sentence ordering methods are summarized into four categories.

1) Clustering and similarity-based methods. These methods cluster the sentences into different clusters. For each cluster, sentences are ordered by greedy selection for maximizing similarity between adjacent sentences [1].

2) Domain dependent-based methods. For example, the entity dependent relations are learned from their corresponding domain to guide sentence ordering 
[2]. Hidden Markov model learns topic shift and keywords shift to guide sentence ordering [3]. The entity-based method combines with Hidden Markov model to form a hybrid model $[4,5,6]$.

3) Syntactic rules-based methods. The structure of text is assumed as a tree. The set of semantic units are linked through rhetorical or discourse relation, where coherent texts will be characterized by valid text plans that satisfies most constraints[7]. The other discourse relations are investigated as well[8, $9,10]$.

4) Other methods. For example, textual complexity-based sentence ordering method[11]; semantic link-based coherence measurement[12, 13]; publication date and temporal cues-based method[14].

Herein, the limitations of the above methods are summarized as follows,

1) Weak ability in semantic association representation and inference. Similaritybased methods only consider the keyword overlaps between sentences and thus lose much association semantics.

40 2) little attention paid on cognitive logical structures. Most existing sentence ordering methods pay less attention on some basic cognitive logical structures such as induction or deduction.

3) Weak foundation of cognitive mechanism. Some researchers have proposed some coherence measurement methods which overemphasize on computation other than positive effect of cognitive process.

4) Non-automatic construction. Many inflexibility syntactic rules are used in sentence ordering and thus cause intractable computation.

To overcome the above limitations, we propose three cognitive logical structures and build markov random fields conditioned on cognitive logical structures for semantic representation and inference. What is more, we develop sound cognitive mechanism for fast sentence ordering. The following properties of our model are different from the above existing methods,

1) Keeping semantic association and inference by markov random filed. Our 
model develops semantic markov random field by which the association semantics is represented and inference is made;

2) Incorporating cognitive logical structures into markov random filed. Our model proposes three cognitive logical structures which include different transactions from which different association relations are mined;

3) Providing sound cognitive mechanism working on sentence ordering. Our model simulates human cognitive process and develops machine oriented sentence ordering model.

The remainders of this paper are organized as follows. Section 2 introduces the preliminary knowledge and basic idea of our model. Section 3 proposes our cognitive memory-inspired sentence ordering model. Section 4 proposes cognitive logical structure learning method; section 5 proposes spreading and activation computation method; section 6 proposes cognitive decision making method; section 7 gives the sentence ordering procedure; section 8 reports experiments. Section 9 makes conclusion.

\section{Preliminary Knowledge and Basic Idea}

Before proposing our sentence ordering model, we propose preliminary knowledge about cognitive logical structure and markov random field for semantic representation and inference. Then we introduce the basic idea of sentence ordering in human beings' discourse process.

\subsection{Preliminary Knowledge}

75

As the coherent knowledge is formalized by double-process of induction and deduction or abstraction and concretization[15, 16, 17, 18]. Both deductive and inductive arguments occur frequently and naturally where the both forms of reasoning can be equally compelling and persuasive[19]. Therefore, we define first two types of cognitive logical structures to capture the above two forms of reasoning. Besides, we further add another type of cognitive logical structure since we found that some author's points are illustrated one by one in text. 
To summarize, we define three most basic types of cognitive logical structure.

It is of course that different combinations of the defined three basic types will generate different mixture structures.

\section{Definition 1:Cognitive Logical Structures, CLS}

$$
C L S=\left\{\aleph_{S P I F}, \aleph_{I P S F}, \aleph_{I O O}\right\}
$$

where:

1. $\aleph_{S P I F}$ denotes a structure that Summary is Prior and Illustration Follows, where text first proposes a summary of main argument and then illustrates supporting details.

2. $\aleph_{I P S F}$ denotes a structure that Illustration is Prior and Summary Follows, where text first illustrates some supporting details and then a summary of main argument follows.

3. $\aleph_{I O O}$ denotes a structure that Illustration is One by One, where author's points are illustrated one by one in text.

Generally, the sentence ordering is closely related to cognitive logical struccorrespond to different sentence orders. For example, there are three unordered sentences:

- $s_{1}$ : Tom breaks a cup.

- $s_{2}$ : The cup is new.

- $s_{3}$ : Tom is careless.

Conditioned on different cognitive logical structures, sentence orders are different as shown in Table 1.

Table 1 shows the sentence relation under different cognitive logical structures, where $s_{i}$ denotes sentence $i$ in sentence set and $s_{(i)}$ denotes the $i^{t h}$ sentence in sentence order; For $\aleph_{S P I F}, s_{(1)}$ is strongly associated with $s_{(2)}$ and $s_{(3)}$; For $\aleph_{I P S F}, s_{(1)}$ and $s_{(2)}$ are strongly associated with $s_{(3)}$; For $\aleph_{I O O}, s_{(1)}$ is strongly associated with $s_{(2)}$, and $s_{(2)}$ is strongly associated with $s_{(3)}$. 
Table 1: three cognitive logical structures and their corresponding sentence orders

\begin{tabular}{|c|c|c|c|}
\hline $\begin{array}{l}\text { Cognitive Logical } \\
\text { Structures } \\
(\mathrm{CLS})\end{array}$ & $\begin{array}{l}\text { Summary is Prior } \\
\text { Illustration Follows } \\
\qquad\left(\aleph_{S P I F}\right)\end{array}$ & $\begin{array}{l}\text { Illustration is Prior } \\
\text { Summary Follows } \\
\left(\aleph_{I P S F}\right)\end{array}$ & $\begin{array}{l}\text { Illustration is } \\
\text { One by One } \\
\left(\aleph_{I O O}\right)\end{array}$ \\
\hline \multirow{2}{*}{ sentence order } & \multicolumn{3}{|c|}{$S_{(1: 3)}=\left(s_{(1)}, s_{(2)}, s_{(3)}\right)$} \\
\hline & $\left(s_{1}, s_{2}, s_{3}\right)$ & $\left(s_{2}, s_{3}, s_{1}\right)$ & $\left(s_{2}, s_{1}, s_{3}\right)$ \\
\hline$s_{(1)}$ & Tom breaks a cup. & The cup is new. & Tom is careless. \\
\hline$s_{(2)}$ & The cup is new. & Tom is careless. & Tom breaks a cup. \\
\hline$s_{(3)}$ & Tom is careless. & Tom breaks a cup. & The cup is new. \\
\hline \multirow{2}{*}{ Sentences association } & $s_{(1)} \rightarrow s_{(2)}$ & $s_{(1)} \rightarrow s_{(3)}$ & $s_{(1)} \rightarrow s_{(2)}$ \\
\hline & $s_{(1)} \rightarrow s_{(3)}$ & $s_{(2)} \rightarrow s_{(3)}$ & $s_{(2)} \rightarrow s_{(3)}$ \\
\hline
\end{tabular}

Semantic representation and inference is an important issue for sentence ordering. Markov random field is a basic undirected probabilistic graphic model with outstanding abilities in semantic representation and inference. Herein, we adopt Markov random field for semantic representation and inference in sentence ordering $[20,21]$. Conditioned on different cognitive structures, semantic markov random field is represented as follows.

Definition 2: Semantic Markov Random Field, SMRF $_{\aleph}$

$$
S M R F_{\aleph}=<G, P(X)>
$$

where $G=<X, E>$ is an undirected graph; $X$ denotes a random variable set and $E$ denotes a dependence relation set between $X$; $\aleph$ denotes a cognitive logical structures( $\aleph \in \mathrm{CLS}) ; P$ denotes a joint probability distribution over $X$, which is calculated by:

$$
P(X)=\mu_{0} \Pi_{c_{i} \in C} \Psi\left(X_{c_{i}}\right)
$$

$c_{i}$ denotes a maximal clique which has no more vertices to add for remaining ${ }_{120}$ fully-connected subgraph; $C=\left\{c_{1}, c_{2}, \ldots\right\}$ denotes a maximal clique set; $X_{c_{i}}$ 
is a maximal clique variable of $c_{i} ; \Psi\left(X_{c_{i}}\right)$ is a non-negative potential function over $X_{c_{i}} ; \mu_{0}=\frac{1}{\sum_{x} \prod_{x_{c_{i}} \in C} \Psi\left(x_{c_{i}}\right)}$ is a normalization factor.

In human being's concept learning and reasoning, Luo et al proposed 2degree hypothesis that human beings make inference only by some low-degree association relation $\left\{\Phi_{k} \mid 0 \leq k \leq 2\right\}$ which occupy more than $90 \%$ of the whole association relations since $\left\{\Phi_{k} \mid 2<k\right\}$ only occupy less than $10 \%$. Inspired above theory, we use 2-degree hypothesis as the constrains on markov random field for simulating semantic representation and inference as human do.

Definition 3:Power Series Representation, $P S R_{\aleph}$

$$
\begin{gathered}
P S R_{\aleph}=\left\{\Phi_{k} \mid 0 \leq k \leq 2\right\} \\
\Phi_{k}=\left\{\phi_{k, i}\right\}
\end{gathered}
$$

where $\aleph$ denotes a specialized cognitive logical structure $(\aleph \in C L S) ; \Phi_{k}$ denotes a k-degree association relation set; $\phi_{k, i}=w_{0}^{(k, i)}, w_{1}^{(k, i)}, \ldots, w_{k-1}^{(k, i)} \rightarrow w_{k}^{(k, i)}$ is a k-degree association relation index i in $\Phi_{k}$ for which $w_{i}^{(k, i)}$ denotes a word and whose support value is calculated by,

$$
\sup _{\aleph}\left(\phi_{k, i}\right)=\frac{\sum_{\operatorname{tran}_{j} \in \mathbb{K}} I\left(\phi_{k, i} \mid \operatorname{tran}_{j}\right)}{\sum_{\operatorname{tran}_{j} \in \mathbb{K}} I\left(\operatorname{tran}_{j} \mid \operatorname{tran}_{j}\right)}
$$

or

$$
\sup _{\aleph}\left(\phi_{k, i}\right) \approx \Sigma_{x} P\left(x \mid S M R F_{\aleph}\right) I\left(\phi_{k, i} \mid x\right)
$$

\subsection{Basic Idea}

Sentence ordering task can be regarded as restrictive writing, since the order of given sentences is unknown and the human beings' task is to order these sentences for well semantic coherence. Based on cognitive process of writing $[22,23,24]$, writing can be characterized as a "journey of discovery".

Fig. 1 shows sentence ordering process in restrictive writing[22], which spans on three memory modules: 1)long term memory, 2)short term memory and 3)working memory as follows. 


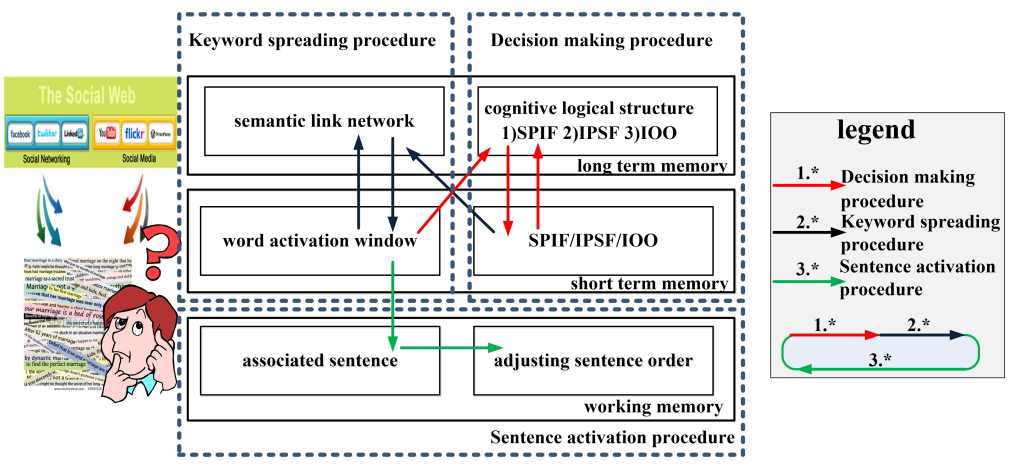

Figure 1: sentence ordering process works on three memory modules in restrictive writing

1) Long term memory contains the relatively stable entities and relations. These entities and relations are stored by semantic link network. Besides, some cognitive logical structures are stored in this module. Keywords can spread towards different directions on semantic link network conditioned on different cognitive logical structures.

2) Short term memory contains a cognitive logical structure window for containing a activated structure and a word activation window with $m$ activated keywords $(5 \leq m \leq 9)$ since Millers Law pointed out that the number of objects an human averagely hold in short term memory is seven, plus or minus two[25].

3) Working memory contains a newly generated sentence closely associated with activated keywords and a sentence order to be adjusted by spreading activation process. The spreading activation process will link all the unordered sentences toward well semantic coherence.

The dynamical ordering procedures works on different modules including:

1) Cognitive logical structure and association semantic construction procedure. The cognitive logical structures and semantic link network lay the knowledge foundation for semantic inference and computation of sentence ordering since sentences will been ordered differently based on different cognitive cognitive 
structures and different semantic link networks.

2) Decision making procedure decides which cognitive logical structure to guild spreading activation, which is conditioned on the cognitive logical structure and keywords in keyword activation window in previous time since human beings often decide sentence structure basic on the previous sentence structure and latest writing content. For example, if previous sentence structure is that summarization followed with illustration and latest writing content are summarization, and then next structure is illustration with higher probability.

3) Spreading activation procedure on semantic link network, which includes keyword spreading and sentence activation. Keyword spreading procedure activates some keywords into keyword activation window conditioned on the current activated keywords and current cognitive logical structure; Sentence activation procedure activates a sentence which is closely associated with the activated keywords in keyword activation window and adds the sentence into the sentence order.

If all the above procedures have been constructed or learned, a human being generates a sentence order by the following procedures when he/she faces some unordered sentences.

175 1) Given some keywords to be developed, he/she makes a decision on which cognitive logical structure is used to develop these keywords by other keywords before generating a new sentence. For example, induction or deduction.

2) Knowing cognitive logical structure, he/she does keyword spreading from the given keywords to other relevant keywords on semantic link network, where different cognitive logical structures correspond different spreading directions.

3) Given associated keywords in short memory, he/she can activate the corresponding sentence which is close with the these associated keywords.

The above steps loop until all the unordered sentences are linked in coherent 185 way. 


\section{Proposed Model}

To simulate the human beings' cognitive process in sentence ordering, we propose cognitive memory inspired sentence ordering model.

Definition 4: Cognitive Memory-inspired Sentence Ordering Model, CM-SOM

$$
C M-S O M \models\left(W_{(t)}, \aleph_{(t)}, S M R F_{\aleph_{(t)}}, S_{\{1: T\}}, S_{(1: t)}, R, O P\right) \quad(t \leq T)
$$

where $\mathrm{t}$ denote the time when adding the $t^{t h}$ sentence; $W_{(t)}$ denotes a keyword activation window at $t^{t h}$ sentences; $\aleph_{(t)}$ denotes a cognitive logical structure $\left(\aleph_{(t)} \in C L S\right) ; S M R F_{\aleph_{(t)}}$ denotes semantic markov random field under $\aleph_{(t)} ; S_{\{1: T\}}=\left\{s_{1}, s_{2}, \ldots, s_{T}\right\}$ denotes $T$ sentences to be ordered; $S_{(1: t)}=$

$195\left(s_{(1)}, s_{(2)}, \ldots, s_{(t)}\right)$ denotes a sentence order generated by the sentence ordering model $(t \leq T) ; R$ denotes a reward policy for a sentence or a sentence order; $O P=\{\mathfrak{D}, \mathfrak{S}, \mathfrak{A}\}$ denotes different procedures:

1) cognitive logical structure decision making procedure selects the cognitive logical structure conditioned on the former word window and cognitive logical structure by,

$$
\aleph_{(t)} \sim \mathfrak{D}\left(\aleph_{(t-1)}, W_{(t-1)}\right)
$$

2) Keywords spreading procedure spreads keywords influence towards other relevant keywords for updating $W_{(t)}$ conditioned on $W_{(t-1)}$ on $S M R F_{\aleph}$ by,

$$
W_{(t)} \sim \mathfrak{S}\left(W_{(t-1)}, S M R F_{\aleph_{(t)}}\right)
$$

3) Sentence activation procedure uses the keywords in $W_{(t)}$ to activate its relevant sentence $s_{(t)}$ which is added into the sentence order.

$$
s_{(t)} \sim \mathfrak{A}\left(W_{(t)},\left(S_{\{1: T\}}-S_{(1: t-1)}\right)\right)
$$

Fig. 2 outlines the framework of our sentence ordering model. The model begins with unordered sentence order $S_{\{1: T\}}$ and ends with $S_{(1: T)}$ by ordering 200 procedure. 


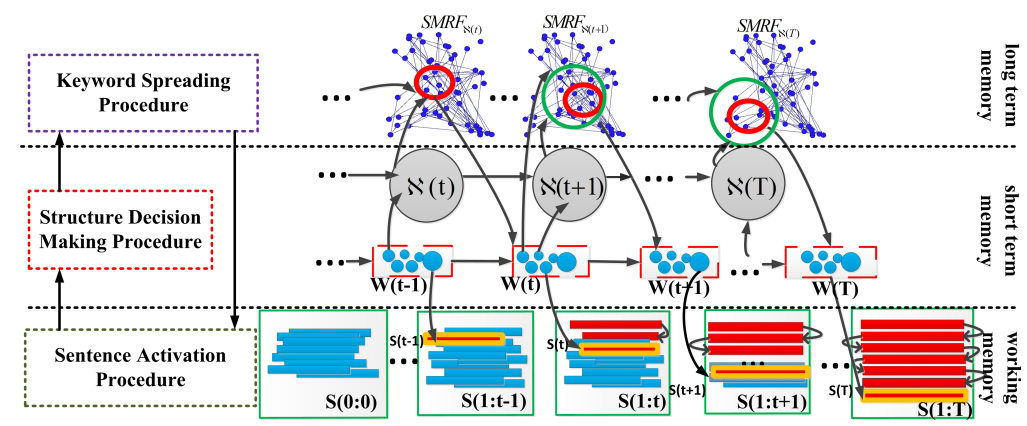

Figure 2: Cognitive memory inspired sentence ordering model

After learning the cognitive memory inspired sentence ordering model and operations on it, a sentence ordering $S_{(1: T)}$ can be drawn by Eq.12,

$$
\begin{gathered}
\left(W_{(1: T)}, \aleph_{(1: T)}, S_{(1: T)}\right) \sim \Pi_{t=1}^{T} P\left(\aleph_{(t)} \sim \mathfrak{D}\left(\aleph_{(t-1)}, W_{(t-1)}\right)\right) \times P\left(W_{(t)} \sim \mathfrak{S}\left(W_{(t-1)}, S M R F_{\aleph_{(t)}}\right)\right. \\
\times P\left(s_{(t)} \sim \mathfrak{A}\left(W_{(t)},\left(S_{\{1: T\}}-S_{(1: t-1)}\right)\right)\right)
\end{gathered}
$$

where $P\left(\aleph_{(t)} \sim \mathfrak{D}\left(\aleph_{(t-1)}, W_{(t-1)}\right)\right)$ denotes the probability of sampling $\aleph_{(t)}$ from $\mathfrak{D}\left(\aleph_{(t-1)}, W_{(t-1)}\right)$, and so on.

A coherence sentence order can be generated by the CM-SOM. As such, we may ask the following question: how can we learn the CM-SOM by machine?

Fig. 3 gives different procedures for learning CM-SOM, which include: 1) cognitive logical structure learning procedure, 2) spreading and activation computation procedure and 3) cognitive decision making procedure.

1) Cognitive logical structure learning procedure. This procedure construct different semantic markov random fields under different cognitive logical structure;

2) Spreading and activation computation procedure. When a cognitive logical structure is selected, this procedure makes keyword spreading and sentence activation based on makov random field;

3) Cognitive decision making learning procedure. This procedure learns the decision making policy for shifting cognitive logical structure. 


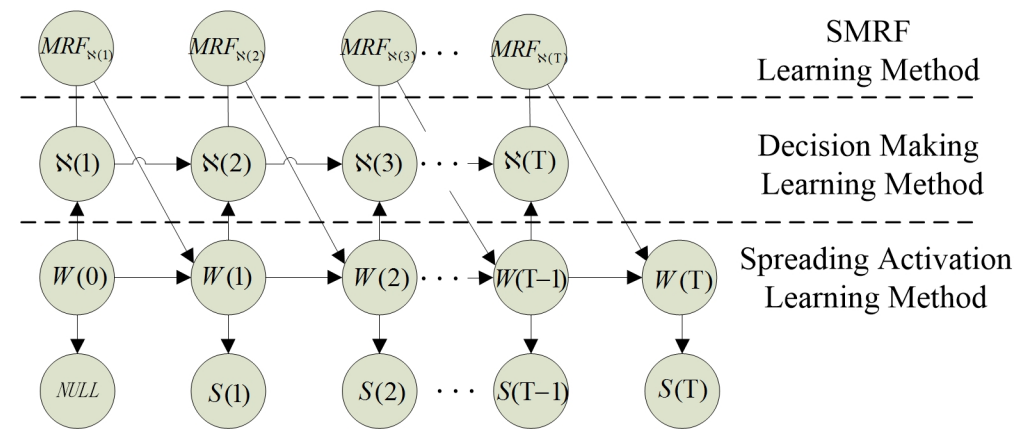

Figure 3: sentence ordering procedure by the proposed sentence ordering model

We list the notations of the above definitions in table 2, which are thoroughly used in this paper.

\section{Cognitive Logical Structure Learning procedure}

Cognitive logical structure learning procedure solves how to learn different cognitive logical structures and learn their corresponding semantic marked random field. these semantic Marked random fields under different cognitive logical structures are global knowledge foundation for sentence ordering since it contains association relations to guide sentence ordering.

\subsection{Cognitive Logical Structure-based Association Representation}

Suppose that a coherent sentence order $S_{(1: T)}=\left(s_{(1)}, s_{(2)}, \ldots, s_{(T)}\right)$ where $s_{(i)}$ denotes the $i^{t h}$ sentence in $S_{(1: T)}$. The sentence order $S_{(1: T)}$ can be convert to different cognitive logical trees which are concrete forms of cognitive logical structure.

Definition 5: Cognitive Logical Tree, $\aleph-t r e e$

$$
\aleph \text {-tree }=(r, \aleph \text {-tree }[i]) \quad 1 \leq i \leq d
$$

where $\aleph \in C L S ; \aleph$-tree consists of a root sentence $r$ and a list of other sub-trees $\aleph$-tree[i]; $d$ is the maximum number of link incident to a sentence node in the cognitive logical tree. 
Table 2: Notations used in the paper

\begin{tabular}{|c|c|}
\hline Symbols & Description \\
\hline$C L S$ & $C L S=\left\{\aleph_{S P I F}, \aleph_{I P S F}, \aleph_{I O O}\right\}$ denotes a set of cognitive logical structure \\
\hline$\aleph$ & $\aleph$ denotes a variable of cognitive logical structure in $C L S$ \\
\hline$\phi_{k, i}$ & $\phi_{k, i}=w_{0}^{(k, i)}, w_{1}^{(k, i)}, \ldots, w_{k-1}^{(k, i)} \rightarrow w_{k}^{(k, i)}$ is a k-degree association relation \\
\hline$\Phi_{k}$ & $\Phi_{k}=\left\{\phi_{k, i}|i<| \Phi_{k} \mid\right\}$ denotes a k-degree association relation set \\
\hline$P S R_{\aleph}$ & $\begin{array}{l}P S R_{\aleph}=\left\{\Phi_{k} \mid 0 \leq k \leq 2\right\} \text { is a set of association relations set under the } \\
\text { structure } \aleph\end{array}$ \\
\hline$X$ & $X=\left\{X_{w_{i}}|0 \leq i \leq| X \mid\right\}$ denotes a random variable set \\
\hline$G=<X, E>$ & $G=\langle X, E\rangle$ is an undirected graph, where $E$ denotes dependence of $X$ \\
\hline$c_{i}$ & $c_{i}$ is a maximal clique which is a full connective sub-graph of $G$ \\
\hline$C$ & $C=c_{i}$ is a set of maximal cliques \\
\hline$X_{c_{i}}$ & a maximal clique random variable of $c_{i}$ \\
\hline$\Psi\left(X_{c_{i}}\right)$ & a non-negative potential function over $X_{c_{i}}$ \\
\hline$\mu_{i}$ & a parameters of Marked random field \\
\hline$P(\widehat{X})$ & marginal probability of $\widehat{X}$ compared with joint probability $P(X)$ \\
\hline$M R F_{\aleph}$ & $M R F_{\aleph}=<G, P(X)>$ denotes Marked random field under the structure $\aleph$ \\
\hline$s_{i}$ & $s_{i}$ denotes the sentence with id $i$ \\
\hline$S_{\{1: T\}}$ & $S_{\{1: T\}}=\left\{s_{1}, s_{2}, \ldots, s_{T}\right\}$ denotes $T$ sentences to be ordered $(t \leq T)$ \\
\hline$s_{(i)}$ & $s_{(i)}$ denotes the $i^{t h}$ sentence \\
\hline$S_{(1: t)}$ & $S_{(1: t)}=\left(s_{(1)}, s_{(2)}, \ldots, s_{(t)}\right)$ denotes a sentence order with $t$ sentences \\
\hline$W_{(t)}$ & $W_{(t)}$ denotes a keyword activation window when selecting the $t^{t h}$ sentence \\
\hline $\mathfrak{D}$ & $\begin{array}{l}\aleph_{(t)} \sim \mathfrak{D}\left(\aleph_{(t-1)}, W_{(t-1)}\right) \text { denotes cognitive logical structure decision making } \\
\text { module }\end{array}$ \\
\hline $\mathfrak{S}$ & $W_{(t)} \sim \mathfrak{S}\left(W_{(t-1)}, S M R F_{\aleph_{(t)}}\right)$ denotes keywords spreading module \\
\hline $\mathfrak{A}$ & $s_{(t)} \sim \mathfrak{A}\left(W_{(t)},\left(S_{\{1: T\}}-S_{(1: t-1)}\right)\right)$ denotes sentence activation module \\
\hline$O P$ & $O P=\{\mathfrak{D}, \mathfrak{S}, \mathfrak{A}\}$ denotes sentence ordering procedure \\
\hline$C M-S O M$ & $\begin{array}{l}C M-S O M \models\left(W_{(t)}, \aleph_{(t)}, S M R F_{\aleph_{(t)}}, S_{\{1: T\}}, S_{(1: t)}, R, O P\right) \\
\text { notes cognitive memory-inspired sentence ordering model }\end{array}$ \\
\hline
\end{tabular}


Table 3: three cognitive logical trees

\begin{tabular}{|c|c|c|c|}
\hline \hline \multirow{2}{*}{ recursive definition } & $\begin{array}{c}\aleph_{S P I F} \text {-tree }= \\
\left(\mathrm{r}, \aleph_{S P I F} \text {-tree[i] }\right)\end{array}$ & $\begin{array}{c}\aleph_{I P S F} \text {-tree }= \\
\left(\mathrm{r}, \aleph_{I P S F} \text {-tree[i] }\right)\end{array}$ & $\begin{array}{c}\aleph_{I O O} \text {-tree }= \\
\left(\mathrm{r}, \aleph_{I O O} \text {-tree[i] }\right)\end{array}$ \\
\hline \multirow{2}{*}{ properties } & $\# r<\# r[i]$ & $\# r>\# r[i]$ & $\# r+1=\# r[i]$ \\
\cline { 2 - 5 } & \multicolumn{3}{|c|}{$\mathrm{r}[\mathrm{i}]$ is the root of subtree $\aleph$-tree[i] } \\
\hline \hline
\end{tabular}

The $\aleph$-tree is a recursive definition which satisfies the following properties in table 3 .

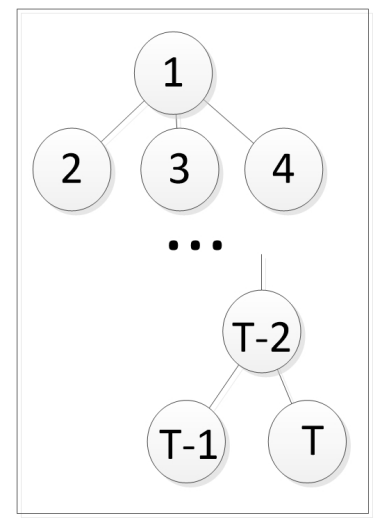

(a) $\aleph_{S P I F-\text { tree }}$

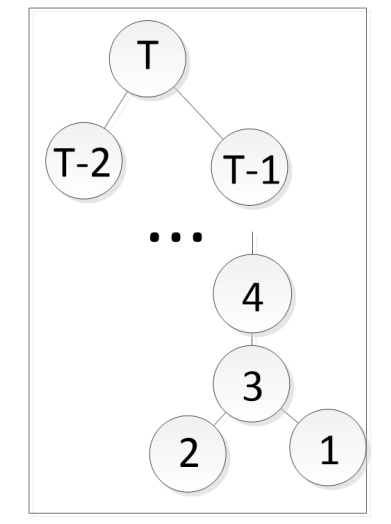

(b) $\aleph_{I P S F}$-tree

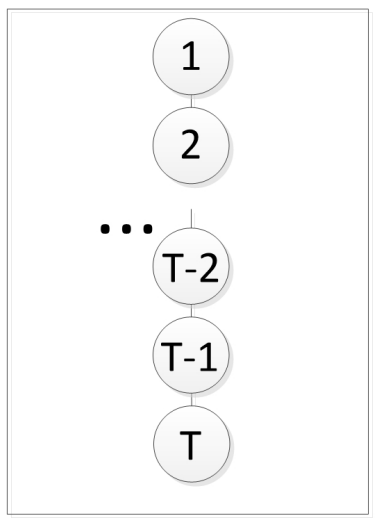

(c) $\aleph_{I O O}$-tree

Figure 4: Cognitive Logical Trees

Supposing manually written texts are well organized by three cognitive logical structures, we learn the three basic cognitive logical trees from them. Given a coherent sentence order $S_{(1: T)}=\left(s_{(1)}, s_{(2)}, \ldots, s_{(T)}\right)$, cognitive logical trees can be learned by algorithm 1 . In algorithm 1 , the sentence $s_{(i)}$ is linked forwards the most relevant one of its previous sentences $s_{(j)}(i-d \leq j<i)$ in $\aleph_{S P I F}$-tree; the sentence $s_{(i)}$ is linked backwards the most relevant one of its following sentences $s_{(j)}(i+d \geq j>i)$ in $\aleph_{I P S F}$-tree; $\aleph_{I O O}$-tree is a special case of $\aleph_{I P S F}$-tree; where adjacent sentences are linked.

Fig. 4 gives three cognitive logical trees of a coherent sentence order $S_{(1: T)}$ where node $i$ corresponds to sentence $s_{(i)}$ in $S_{(1: T)}$. Fig. 4 shows that these 
cognitive logical trees respectively satisfy the properties in table 3 .

Observing these cognitive logical trees in Fig.4, we found that the paths from root node to leaf node are different, which generate different transactions if we regards two adjacent nodes as a transaction in different logical structure trees.

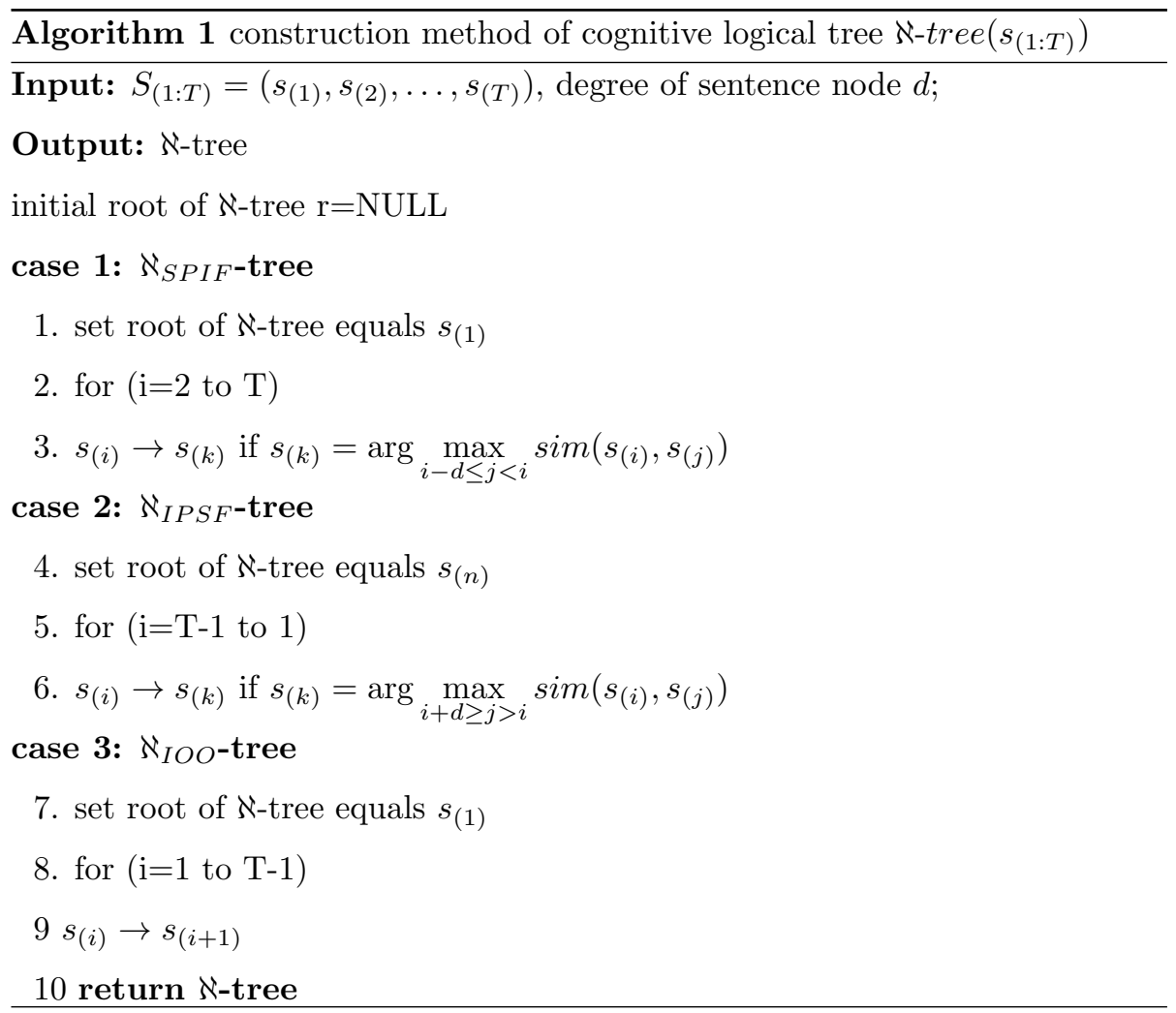

Different transactions generated different cognitive logical trees imply different association relations which mean different keyword spreading direction when semantic representation and inference. For example, $\aleph_{S P I F}$-tree guides keywords to spread from summary keywords to illustrative keywords; $\aleph_{I P S F^{-}}$ tree guides keywords from illustrative keywords to summary keywords; $\aleph_{I O O^{-}}$ tree guides keywords from illustrative keywords to other illustrative ones. Mining association relations from the transactions of different cognitive trees can generate association relations with different keyword spreading directions by algorithm 2 under the $\aleph$-trees $(\aleph \in C L S)$. 
$\overline{\text { Algorithm } 2 \text { construction method of Power Series Representation conditioned }}$ on $\aleph$-tree

Input: $\aleph$-tree $\left(S_{\left(1: T_{1}\right)}\right), \aleph$-tree $\left(S_{\left(1: T_{2}\right)}\right), \ldots, \aleph$-tree $\left(S_{\left(1: T_{n}\right)}\right)$ and sliding window $W$ Output: $P S R_{\aleph}$

initial $P S R_{\aleph}=N U L L$

1. generate transactions by sliding window $W$ on each path from root sentence node to leaf sentence node of $\aleph$-tree

2. mine association relations $\left\{\Phi_{k} \mid 0 \leq k \leq 2\right\}$ from these transactions

3. return $\left\{\Phi_{k} \mid 0 \leq k \leq 2\right\}$

\subsection{Cognitive Logical Structure based-markov Random Field Learning Method}

According to preliminary knowledge, $S M R F_{\aleph}$ model should satisfy the constants of 2-degree hypothesis in human concept learning and reasoning. Learning $S M R F_{\aleph}$ from $P S R_{\aleph}$ is effectiveness since:

1) semantic association consistent. Low-degree association relations in $P S R_{\aleph}$ cover more than $90 \%$ semantic association distribution of $S M R F_{\aleph}$;

2) learning efficiency improvement. Low-degree association relations in $P S R_{\aleph}$ causes small scale probabilistic inference on $S M R F_{\aleph}$.

$S M R F_{\aleph}$ is an undirected probabilistic graphic model and $P S R_{\aleph}$ is an association relation set. Learning $S M R F_{\aleph}$ from $P S R_{\aleph}$ should guarantee structural consistence and semantic consistence by structural collaborative computation and potential value collaborative computation.

\section{Structural collaborative computation}

Structural collaborative computation mainly solves issues: 1) how does $P S R_{\aleph}$ form a graphic structure of $\left.S M R F_{\aleph} ; 2\right)$ what is the parameter structure of $S M R F_{\aleph}$ formed from $P S R_{\aleph}$. Referred to a factor graph theory, we solve the above issues by $1^{\text {st }}-4^{\text {th }}$ steps. For 1 ), we form an undirected graph structure by 
$1^{\text {st }}-2^{\text {nd }}$ steps; For 2), we form the parameter structure by $3^{t h}-4^{t h}$ steps.

1. add a variable $X_{w_{i}}$ in $S M R F_{\aleph}$, only if keywords $w_{i}$ occurs in $P S R_{\aleph}$;

2. link $X_{w_{i}}$ and $X_{w_{j}}$ in $S M R F_{\aleph}$, only if $w_{i}$ and $w_{j}$ co-occur in an association relations in $P S R_{\aleph}$;

3. map each association relation into a maximal clique, only if the keywords in association relations are contained in this maximal clique;

4. set the parameter structure for each maximal clique variable by,

$$
\Psi_{X_{c_{i}}}=\Pi_{\phi_{i} \in\left\{\Phi_{k} \mid 1 \leq k \leq 2\right\}} \mu_{i}^{I\left(\phi_{i} \mid X_{c_{i}}\right)}
$$

where $X_{c_{i}}$ denotes a maximal clique variable; $\mu_{i}$ is a parameter of a semantic association relation $\phi_{i}$ in $P S R_{\aleph} ; I\left(\phi_{i} \mid X_{c_{i}}\right)$ is an indicator function whose outcome is 1 when association relation $\phi_{i}$ is consistent with the maximal clique variable $X_{c_{i}}, 0$ otherwise.

According to $1^{\text {st }}-4^{\text {th }}$ steps, we propose a structural collaborative algorithm 3.

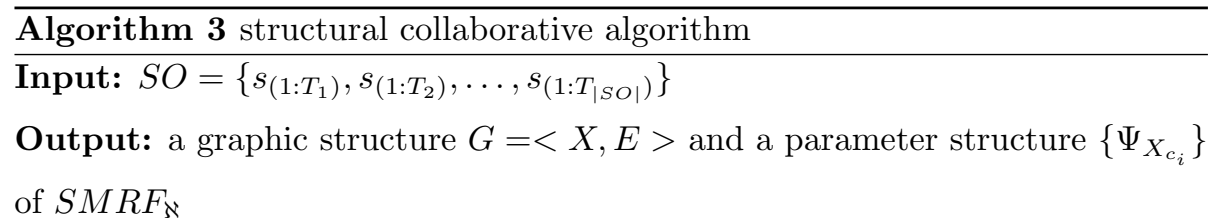

1. construction cognitive logical trees by algorithm 1

2. mine $P S R_{\aleph}$ by algorithm 2

3. form $G=<X, E>$ by $1^{\text {st }}-2^{\text {nd }}$ steps

4. form $\left\{\Psi_{X_{c_{i}}}\right\}$ by $3^{t h}-4^{t h}$ steps

5. return $G=<X, E>$ and $\left\{\Psi_{X_{c_{i}}}\right\}$

\section{Potential value collaborative computation}

To guarantee semantic association consistence between $P S R_{\aleph}$ and $S M R F_{\aleph}$, we propose potential value collaborative computation which learns potential values of $S M R F_{\aleph}$ from $P S R_{\aleph}$. It is easily found that $\sup \left(\phi_{k, i} \mid \aleph\right)$ defined as Eq. 6 is 
unbiased estimation of marginal probability $P\left(\phi_{k, i}\right)$ defined as Eq.7 by,

$$
\left|\Sigma_{x} P\left(x \mid S M R F_{\aleph}\right) I\left(\phi_{k, i} \mid x\right)-\sup _{\aleph}\left(\phi_{k, i}\right)\right|<\varepsilon
$$

295

300

$$
\mu_{i}^{t+1}=\mu_{i}^{t} \times \frac{\sup \left(\phi_{i}\right) \times\left(1-P^{t}\left(\phi_{i}\right)\right)}{P^{t}\left(\phi_{i}\right) \times\left(1-\sup \left(\phi_{i}\right)\right)}
$$

We propose a potential value collaborative algorithm 4. A common issue among

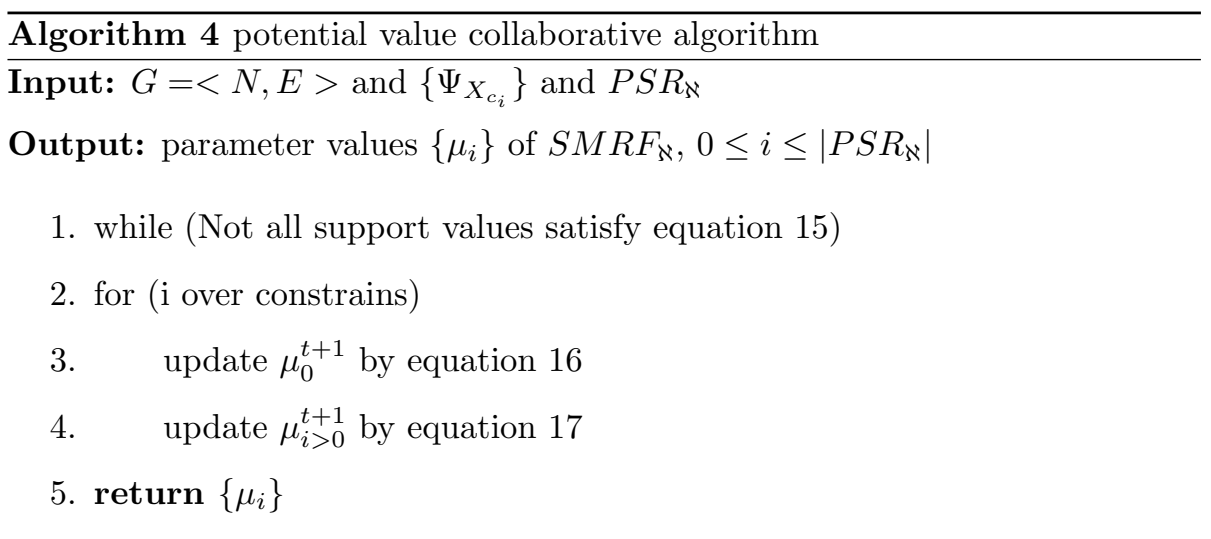

equations $15-17$ is to calculate the marginal probability of $S M R F_{\aleph}$. To calculate marginal probability, we adopt the junction tree algorithm which is a general probabilistic inference framework for calculating joint probability, marginal probability and condition probability [30]. 


\section{Spreading and Activation Computation}

Given $S M R F_{\aleph}=<G, P(X)>$, marginal and conditional probabilities are easily calculated by Eq. 18 and Eq. 19.

$$
P\left(x_{A} \mid S M R F_{\aleph}\right)=\Sigma_{x} P\left(x \mid S M R F_{\aleph}\right) I\left(x_{A} \mid x\right)
$$

where $x_{A}$ denotes a subset of random variables $x ; I\left(x_{A} \mid x\right)$ is an indicator func-

310

$$
P\left(x_{A} \mid x_{B}, S M R F_{\aleph}\right)=\frac{\Sigma_{x} P\left(x \mid S M R F_{\aleph}\right) I\left(x_{A B} \mid x\right)}{\Sigma_{x} P\left(x \mid S M R F_{\aleph}\right) I\left(x_{B} \mid x\right)}
$$

where $x_{A}$ and $x_{B}$ denote a subset of random variables $x$.

\subsection{Keyword Spreading Based Computation}

Given $W_{(t-1)}$ and $\aleph_{(t)}$, sentence ordering model can obtain $W_{(t)}$ by keywords 315 spreading process as Eq.20

$$
W_{(t)} \sim P\left(W_{(t)} \mid W_{(t-1)}, S M R F_{\aleph_{(t)}}\right)
$$

The above process are conducted by sampling from $S M R F_{\aleph_{(t)}}$, however $W_{(t)}$ corresponds to many combination states since any keyword may occur in $W_{(t)}$. To reduce the keyword combinations, we constrain the keywords appear in $W_{(t)}$ only if these keywords co-occur at least one sentence. $W_{(t)}^{s}$ denotes candidate keywords which can appear in $W_{(t)}$ and $P\left(W_{(t)} \mid W_{(t-1)}, S M R F_{\aleph_{t}}\right)$ is calculated by,

$P\left(W_{(t)}^{s} \mid W_{(t-1)}, S M R F_{\aleph_{(t)}}\right)=1-E X P\left(\sum_{w_{i} \in W_{(t)}^{s} \subseteq s_{i} \in \bar{S}} \log \left(1-p\left(w_{i} \mid W_{(t-1)}, S M R F_{\aleph_{(t)}}\right)\right)\right)$

where $\bar{S}$ denotes the sentences to be order; $W_{(t)}^{s}$ denotes the keywords co-occur at least one sentence of $\bar{S}$.

The keywords of $W_{(t)}$ complete sampling when the keywords in $W_{(t)}^{s}$ maximize the probabilistic value of $P\left(W_{(t)} \mid W_{(t-1)}, S M R F_{\aleph_{(t)}}\right)$ by,

$$
W_{(t)}=\arg \max _{W_{(t)}^{s}} P\left(W_{(t)}^{s} \mid W_{(t-1)}, S M R F_{\aleph_{(t)}}\right)
$$


where $W_{(t)}$ denotes the sampled keywords at $t$ time.

\subsection{Sentence Activation Based Computation}

320

Given $W_{(t)}, s_{i}$ is be activated with probabilistic value of $P\left(s_{i} \mid W_{(t)}, S M R F_{\aleph_{(t)}}\right)$ by Eq.23,

$$
P\left(s_{i} \mid W_{(t)}, S M R F_{\aleph_{(t)}}\right)=1-E X P\left(\sum_{w_{i} \in s_{i} \in \bar{S}} \log \left(1-p\left(w_{i} \mid W_{(t)}, S M R F_{\aleph_{(t)}}\right)\right)\right)
$$

where $\bar{S}$ denotes the sentences to be order; $w_{i}$ denotes a keyword occurs in $s_{i}$ of $\bar{S}$.

$s_{(t)}$ complete sampling when the sentence $s_{i}$ maximizes the probabilistic value of $P\left(s_{i} \mid W_{(t)}, S M R F_{\aleph_{(t)}}\right)$ by,

$$
s_{(t)}=\arg \max _{s_{i}} P\left(s_{i} \mid W_{(t)}, S M R F_{\aleph_{(t)}}\right)
$$

325

\section{Cognitive Decision Learning Module}

Cognitive decision making is a core module of cognitive memory-inspired sentence ordering model(CM-SOM) since: 1) cognitive decision making module decides which cognitive logical structure and 2) different cognitive logical policy for cognitive logical structure decision making is an important issue for the cognitive decision learning model since such policy decides how to the logical structures shift during sentence ordering.

\subsection{Cognitive Logical Structure Decision Making Policy}

Structure decision making policy decides which cognitive logical structure $\aleph_{(t-1)}$ is selected to develop a new sentence conditioned on structure $\aleph_{(t-1)}$ and activated keyword window $W_{(t-1)}$. 


\section{Definition 6: Structure Decision Making Policy, $r_{t}$}

$\mathfrak{D}=\left\{Q\left(\aleph_{(t-1)}, d_{W_{(t-1)}}, \aleph_{t}\right) \mid p\left(\aleph_{(t)} \sim \mathfrak{D}\left(\aleph_{(t-1)}, W_{(t-1)}\right)\right) \propto Q\left(\aleph_{(t-1)}, d_{W_{(t-1)}}, \aleph_{t}\right)\right\}$

where $d_{W_{(t-1)}}$ denotes power of $W_{(t-1)}$ to make shift from one cognitive logical structures to other one. $d_{W_{(t-1)}}$ values can be calculated flexibly by some measurable properties of keywords in $W_{(t-1)}$, such as the frequency or the degree of keywords.

In CM-SOM, the driving force for generating a sentence order is decision making module of cognitive logical structure since decision making module is the precondition of other modules.

Suppose a sentence order $S_{(1: t)}$ is generated by ordering procedure of CMSOM by Eq.12, the driving force is defined by,

Definition 7: Structure-Driven Knowledge Flow, $h$

$$
\begin{gathered}
h=\left(\aleph_{(1)}, W_{(1)}, \ldots, \aleph_{|h|}, W_{|h|}\right) \\
h_{(t)} \subseteq H^{t} \subset H^{*}
\end{gathered}
$$

where $t$ is the length of $S_{(1: t)} ; H^{t}=\{h:|h|=t\}$ is a set of structure-driven knowledge flow with length $t ; H^{*}=\bigcup_{i=0}^{\infty} H^{t}$ denotes a set of structure-driven knowledge flow with any length $1<t<\infty$.

The probability of $h$ with length $t$ to be generate by CM-SOM is calculated by,

$$
\begin{aligned}
p(h, t \mid \mathfrak{D})= & p\left(\aleph_{(0)}\right) p\left(W_{(1)}\right)\left[\Pi_{i=1}^{t} p\left(\aleph_{(i)} \sim \mathfrak{D}\left(\aleph_{(i-1)}, W_{(i-1)}\right)\right)\right. \\
& \left.p\left(W_{(i)} \sim \mathfrak{S}\left(W_{(i-1)}, \aleph_{(i)}\right)\right)\right] I(|h|=t) p(t)
\end{aligned}
$$

where $I(|h|=t)=1$ if $|h|=t, 0$ otherwise; $p(t)$ is a prior over length $t$.

In cognitive memory inspired sentence ordering model, any $h$ generated by the model will obtain reward value $r_{t} \quad 0<t \leq \infty$ which is defined by,

Definition 8: Reward of Structure-Driven Knowledge Flow, $r_{t}$

$$
r_{t}=p(R=1 \mid h, t)
$$


where $r_{t} \in[0,1] ; p(R=1 \mid h, t)$ is probability equaling to $r_{t}$.

The $r_{t}$ value can be calculated flexibly. For example, we can calculate it by the relevance value of $\left(W_{(t)}, s_{(t)}\right)$ or the rank correlation coefficient of $\left(S_{(1: t)}, S_{(1: t)}^{*}\right)$ where $S_{(1: t)}^{*}$ denotes standard sentence order which have be given.

The expected reward of a decision making policy is calculated by,

$$
\begin{aligned}
V(\mathfrak{D}, R) & =E_{t}\left[r_{t} ; \mathfrak{D}\right] \\
& =E_{h, t}[p(R=1 \mid h, t) ; \mathfrak{D}] \\
& =\sum_{h, t} p(R=1 \mid h, t) p(h, t ; \mathfrak{D}) \\
& =\sum_{h, T, t} p(R=1 \mid h, t) p(h, T, t ; \mathfrak{D}) \\
& =\sum_{h, T, t} p(R=1, T, t, h ; \mathfrak{D})
\end{aligned}
$$

where $0<t \leq \infty ; h \in H^{*} ; p(h, T, t \mid \mathfrak{D})$ is calculated by,

$$
p(h, T, t \mid \mathfrak{D})=a(T) b(t) p(h \mid t ; \mathfrak{D})
$$

where $a(T)=(1-\alpha) \alpha^{t} ; b(t)=(1-\beta / \alpha)(\beta / \alpha)^{t} ; \beta<\alpha<1$.

The log probability of structure-driven knowledge flow $h^{T, t}$ is calculated by,

$$
\begin{aligned}
\log p\left(h^{T, t} \mid t ; \mathfrak{D}\right) & =\text { const. }+\log \left(\Pi_{i=1}^{t} p\left(W_{(i)} \sim \mathfrak{S}\left(W_{(i-1)}, \aleph_{(i)}\right)\right) p\left(\aleph_{(i)} \sim \mathfrak{D}\left(\aleph_{(i-1)}, W_{(i-1)}\right)\right)\right. \\
& =\text { const. }+\sum_{\aleph, W, \aleph^{\prime}} c_{\aleph, W, \aleph^{\prime}} \log \left(p\left(\aleph^{\prime} \sim \mathfrak{D}(\aleph, W)\right)\right)
\end{aligned}
$$

where $c_{\aleph, W, \aleph^{\prime}}$ denotes the number of occurrences of triple in $\aleph, W, \aleph^{\prime}$ in $h^{T, t}$.

\subsection{Optimal Decision Making Policy Learning Method}

An optimal decision making policy of CM-SOM is learned by maximizing rewards as Eq.33 and CM-SOM can generate a coherent sentence order by spreading process as Eq.12.

$$
\mathfrak{D}^{*}=\arg \max _{\mathfrak{D}} V(\mathfrak{D}, R \mid S O)
$$

We learn the cognitive logical decision process from the coherent sentence order set by maximizing reward value. Inspired by Monte-carlo-based algorithm[31, 
32] and Stochastic Approximation $\operatorname{EM}(\mathrm{SAEM})$ algorithm[33, 34], we learn $\mathfrak{D}^{*}$ by EM algorithm since $V(\mathfrak{D})$ can be regraded as likelihood of joint distribution $p(R, T, t, h ; \mathfrak{D})$ where latent variables $t, T$ and the structure-driven knowledge flow $h$ are marginalized out by Eq.34,

$$
\begin{aligned}
l(\mathfrak{D} ; R) & =\log \sum_{T, t, h} p(R, T, t, h ; \mathfrak{D}) \\
& =\log \sum_{T, t, h} q(T, t, h \mid R) \frac{p(R, T, t, h ; \mathfrak{D})}{q(T, t, h \mid R)} \\
& \geq \sum_{T, t, h} q(T, t, h \mid R) \log \frac{p(R, T, t, h ; \mathfrak{D})}{q(T, t, h \mid R)} \\
& =\mathcal{L}(q, \mathfrak{D})
\end{aligned}
$$

where $\mathcal{L}(q, \mathfrak{D})$ is the lower bound for $l(\mathfrak{D} ; R) . \mathcal{L}(q, \mathfrak{D})$ can be maximized by EM algorithm.

Inspired by the approximation EM algorithm in [34], we develop the algorithm as follows.

1. In E-step, we sample $h^{t, T}$ from the posterior over the latent variables given the observed data $R$ and for given parameters $\mathfrak{D}$ by Eq.35 and assign its weight $w_{h^{T, t}}$ by Eq.36

$$
\begin{gathered}
h^{t, T} \sim p\left(t, h, T \mid R ; \mathfrak{D}_{k-1}\right) \propto a(T) b(t) p\left(h \mid t ; \mathfrak{D}_{k-1}\right) p(R \mid h, T) \\
w_{h^{T, t}}=b(t) p\left(R=1 \mid h^{T, t}, T\right)
\end{gathered}
$$

2. In M-step, we update $\hat{\mathcal{L}}_{k}(\mathfrak{D})$ by Eq.37 and assign $\mathfrak{D}^{*}$ by Eq.38

$$
\begin{aligned}
\hat{\mathcal{L}_{k}}(\mathfrak{D})=\left(1-\alpha_{k}\right) \hat{\mathcal{L}}_{k-1}(\mathfrak{D}) & +\frac{\alpha_{k}}{m} \sum_{i=1}^{m} \frac{1}{\left|h^{i}\right|+1} \sum_{j=0}^{\left|h^{i}\right|} w_{h^{i, j}} \log p\left(h^{i, j} \mid j ; \mathfrak{D}\right) \\
\mathfrak{D}^{*} & =\arg \max _{\mathfrak{D}} \hat{\mathcal{L}}_{k}(\mathfrak{D}) .
\end{aligned}
$$

where $m$ denotes the number of samples. 
By substituting $\log p\left(h^{T, t} \mid t ; \mathfrak{D}\right)$ of Eq. 32 into Eq.37, the $\hat{\mathcal{L}}_{k}(\mathfrak{D})$ can be updated iteratively by,

$\hat{\mathcal{L}}_{k}(\mathfrak{D})=\left(1-\alpha_{k}\right) \hat{\mathcal{L}}_{k-1}(\mathfrak{D})+\frac{\alpha_{k}}{m} \sum_{\aleph, W, \aleph^{\prime}} \log \left(p\left(\aleph^{\prime} \sim \mathfrak{D}(\aleph, W)\right)\right) \sum_{i=1}^{m} \frac{1}{\left|h^{i}\right|+1} \sum_{t=0}^{\left|h^{i}\right|} w_{h^{i, t}} c_{\aleph, W, \aleph^{\prime}}$

The above equation can be converted to Eq.40 and Eq.41.

$$
\begin{gathered}
Q_{k}\left(\aleph, W, \aleph^{\prime}\right)=\left(1-\alpha_{k}\right) Q_{k-1}\left(\aleph, W, \aleph^{\prime}\right)+\frac{\alpha_{k}}{m} \sum_{i=1}^{m} \frac{1}{\left|h^{i}\right|+1} \sum_{t=0}^{\left|h^{i}\right|} w_{h^{i, t}} c_{\aleph, W, \aleph^{\prime}} \\
\hat{\mathcal{L}}_{k}(\mathfrak{D})=\sum_{\aleph, W, \aleph^{\prime}} Q_{k}\left(\aleph, W, \aleph^{\prime}\right) \log \left(p\left(\aleph^{\prime} \sim \mathfrak{D}(\aleph, W)\right)\right)
\end{gathered}
$$

It has been proofed by [34] that the solution of maximization of $\hat{\mathcal{L}}_{k}(\mathfrak{D})$ is $p^{*}\left(\aleph^{\prime} \sim\right.$ $\mathfrak{D}(\aleph, W))$,

$$
p^{*}\left(\aleph^{\prime} \sim \mathfrak{D}(\aleph, W)\right) \propto Q_{k}\left(\aleph, W, \aleph^{\prime}\right)
$$

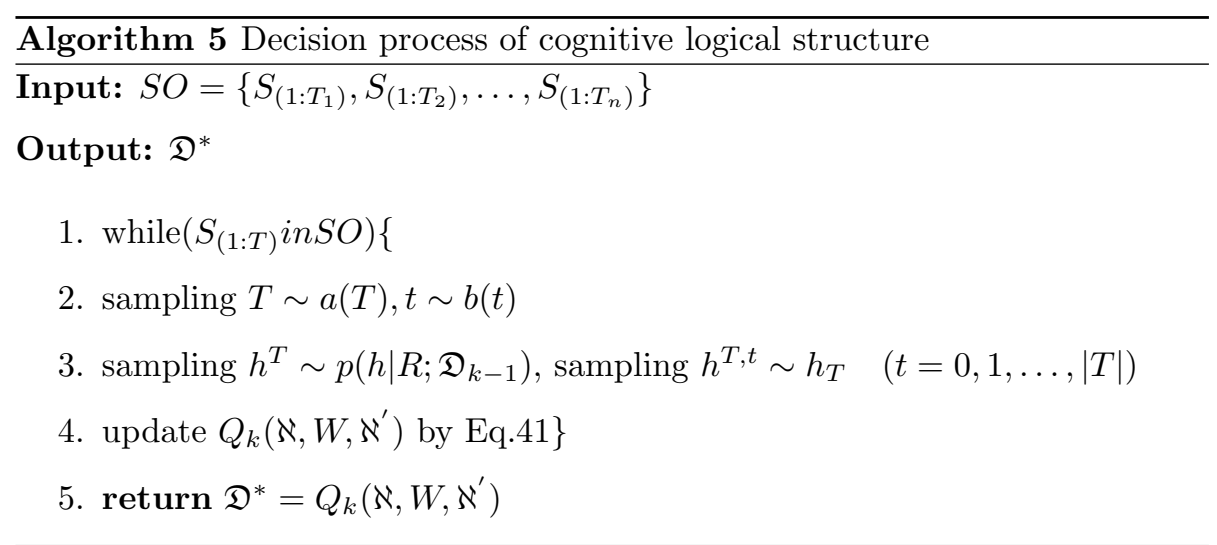

\section{Sentence Ordering Module}

After completing 1) cognitive logical structure learning, 2) spreading activation computation and 3) cognitive structure decision making learning in section has been learned. 
Given a sentence ordering model, CM-SOM defined by Eq.8, CM-SOM can order unordered sentences $S_{\{1: T\}}$ to a sentence order $S_{(1: t)}$ by algorithm 6 .

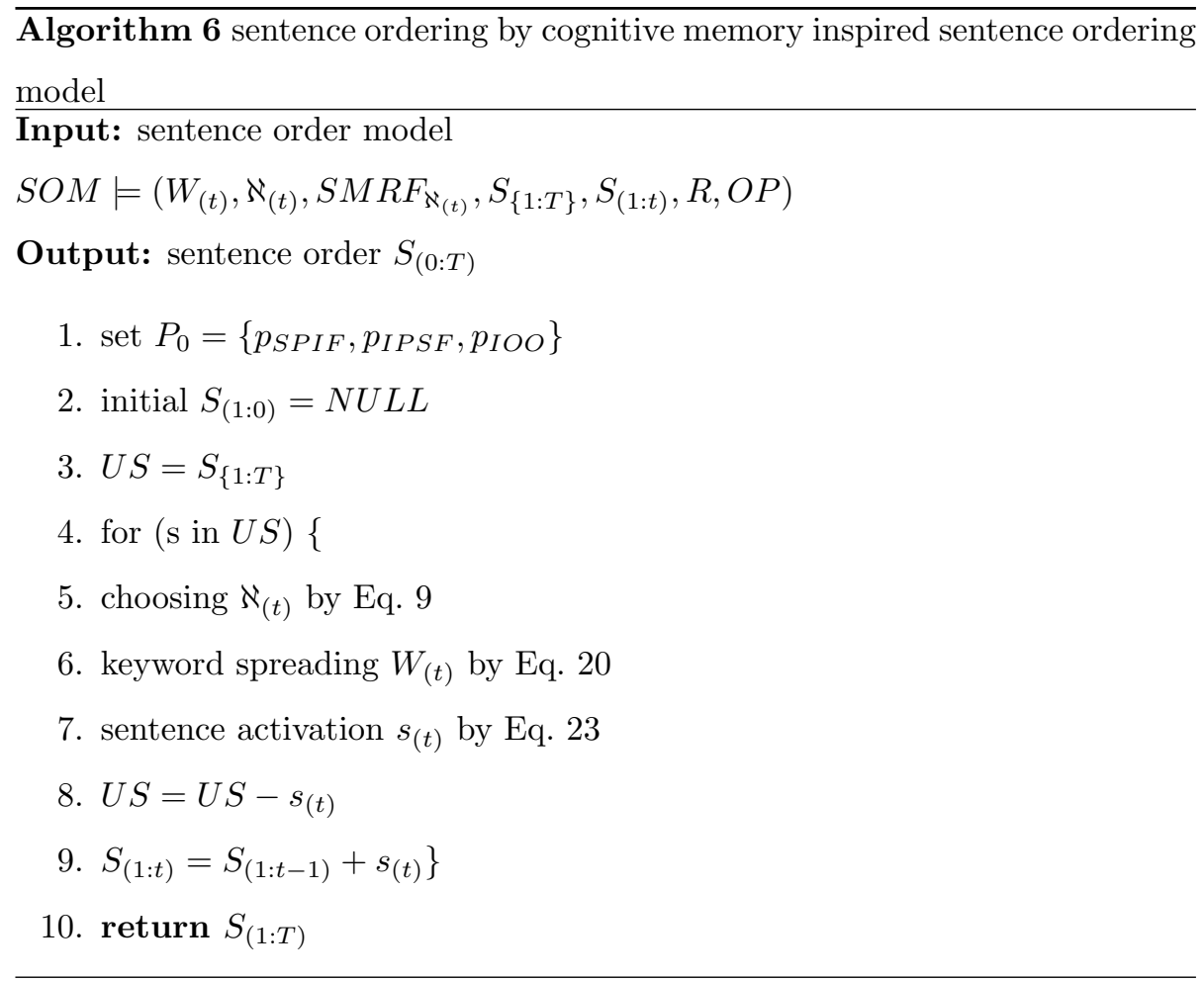

\section{Experiment}

In this section, we mainly conduct some experiments to validate the correctness of our sentence ordering model.

\subsection{Data Set}

We collect 2 datasets which consist of Reuters news as dataset 1 and paper abstracts as dataset 2. Table 4 gives a description of these datasets. In the table, domain data is used to learn logical structure and construct semantic markov random fields under different logical structures; training data is used to learn the decision making policy of cogitative logical structures. Test data is 
used to test the sentence ordering results generated by sentence ordering model. For each dataset, we will randomly select $50 \%$ texts as domain knowledge; $25 \%$ as texts as training data; $25 \%$ texts as test data from each dataset.

- Dataset 1 includes 60,000 pieces of news for which each piece of these news has average 15.67 words and these news are crawled from Reuters website (http://www.reuters.com) from March 2009 to August 2009. These news are about three domains including health, environment, internet;

- Dataset 2 includes 50,000 paper abstracts for which each one has average 13.27 words and these papers are from Association for Computing Machinery-digital Library(http://dl.acm.org/). These papers cover 10 different categories including data mining, machine learning, algorithm and so on

Table 4: Description of datasets

\begin{tabular}{|c|c|c|}
\hline \hline Data Set & Dataset 1 & Dataset 2 \\
\hline Source & routers news & paper abstract \\
\hline \# domain/category & 3 & 10 \\
\hline Avg.\# sentences per text & 15.67 & 13.29 \\
\hline Domain data for learning MRF & $50 \%$ & $50 \%$ \\
\hline Training data for learning CLS & $25 \%$ & $25 \%$ \\
\hline Test data for sentence ordering & $25 \%$ & $25 \%$ \\
\hline Total text number & 60000 & 50000 \\
\hline \hline
\end{tabular}

\subsection{Evaluation Measurement}

Since original news or abstracts are written manually by their authors, we assume these texts have well cognitive logical structures and their original sentence orders are semantic coherent. So the correlation coefficient between the automatically generated sentence order and the original sentence order is used 
to measure the accuracy of sentence ordering modules. Kendall rank correlation coefficient is used to measure rank correlation which is calculated by Eq.43 referred in [2].

$$
k r\left(S_{(1: n)}, S_{(1: n)}^{*}\right)=1-(4 P /(n \times(n-1)))
$$

where $S_{(1: n)}$ denotes an automatically generated sentence order as the benchmark; $S_{(1: n)}^{*}$ denotes the original sentence order as benchmark. $P$ denotes the number of inversion sequence number; $n$ denotes sentence number. $k r \in[0,1]$ : 1 if the generated sentence order is the same with the benchmark; -1 if the sentence order is the reverse of the benchmark; 0 if the two sentence orders are independent with each other.

\subsection{Baseline Method}

To validate efficiency of our sentence ordering model, our sentence ordering model(CM-SOM) is compared with 1) single structure-based sentence ordering models and 2) the state-of-the-art sentence order models in related work.

\section{Single Structure-based Sentence ordering model}

To validate the positive effect of optimal decision making policy and decision making process for sentence ordering, we compare our sentence ordering model with single structure-based sentence ordering models. The single structure based sentence ordering models are listed as follows,

1. sentence ordering model under $\aleph_{S P I F}$, SPIF-SOM. It assumes that sentence ordering process conducts under single cognitive logical structure $\aleph_{S P I F}$ and makes spreading activation on corresponding semantic markov random field $S M R F_{\aleph_{S P I F}}$.

2. sentence ordering model under $\aleph_{I P S F}$, IPSF-SOM. It assumes that sentence ordering process conducts under $\aleph_{I P S F}$ and spreading activation on $S M R F_{\aleph_{I P S F}}$.

3. sentence ordering model in $\aleph_{I O O}$, IOO-SOM. It assumes that sentence ordering process conducts under $\aleph_{I O O}$ and spreading activation on $S M R F_{\aleph_{I O O}}$. 
To validate effectiveness of our sentence ordering model, our sentence ordering model(CM-SOM) is compared with the state-of-the-art sentence ordering models as follows,

1. Sentence-similarity Based Model(SBM)[1]. It assumes that the adjacent sentences of a coherent sentence order have high similarity.

2. Keyword-dependence Based Model(KBM)[2]. It assumes that keywords of adjacent sentences have strong dependance in domain knowledge.

3. Entity-dependence Based Model(EBM)[14]. It assumes that some syntactic role transition patterns often occur between adjacent sentences.

\subsection{Experimental Setup}

To validate efficiency of our sentence ordering model, we design experimental setup as follows.

1. To discover association relations with different cognitive structures, we construct $\aleph_{I P S F} / \aleph_{S P I F} / \aleph_{I O O}$-tree for each text in domain data by algorithm 1 and mine association relations by running algorithm 2 on the unit of whole domain data under different domain respectively .

2. To establish foundation of semantic representation and inference, we construct semantic markov random fields as special association link networks with different cognitive logical structures by running algorithm 3 and algorithm 4 on the unit of whole domain data under different domain respectively.

3. Based on semantic markov random field, we learn policy of decision making by running algorithm 5 on the unit of training data in table 4 .

4. To generate coherent sentence order for each text of test data in table 4 , our sentence ordering model orders sentences by running algorithm 6 on the unit of each article.

5. To validate the efficiency of our model, we compare the results of our sentence ordering model(CM-SOM) with the ones of the baseline methods 
in section 8.3. We evaluate these results by evaluation measurement in section 8.2 .

6. The above comparative experiments are validated by a one-way analysis of variance(ANOVA) since ANOVA show lower significant value Sig. $<$ 0.05 when evaluation measurement show statistically significant difference between different comparative experimental groups.

In decision making algorithm 5, the reward value $r_{t}$ in Eq. 29 and activated window power $d_{W_{(t-1)}}$ in Eq. 25 can be flexibly calculated in specific applications. In our experiments, the values are calculated respectively as shown in table 5 .

Table 5: Details of Experiment

\begin{tabular}{|c|c|c|}
\hline \hline category & $\begin{array}{c}\text { reward function,R: } \\
r_{i}=\end{array}$ & activated window power D: \\
& $d_{W(i)}=$ \\
\hline A & $\frac{s_{(i)} \cap s_{(i)}^{*}}{s_{(i)} \cup s_{(i)}^{*}}$ & $\sum_{k \in W}\lfloor(F(k)-\min F) \times 5 /(\max F-\min F)\rfloor$ \\
\hline B & $\left(k r\left(S_{(1: i)}, S_{(1: i)}^{*}\right)+1\right) / 2$ & $\sum_{k \in W}\lfloor(D(k)-\min D) \times 5 /(\max D-\min D)\rfloor$ \\
\hline
\end{tabular}

According to table 5, we generate different combinations of policy functions and reward functions. Table 6 lists all the combinations which respectively correspond to combination code $0-3$. The following experiments are respectively conducted under such combinations.

Table 6: combinations of policy function and reward function

\begin{tabular}{|l|l|l|}
\hline $\begin{array}{l}\text { combination } \\
\text { code }\end{array}$ & reward function(R) & $\begin{array}{l}\text { activation window } \\
\text { state value }\end{array}$ \\
\hline 0 & A & A \\
\hline 1 & A & B \\
\hline 2 & B & A \\
\hline 3 & B & B \\
\hline \hline
\end{tabular}


Given the reward function and activation window state value above, we can conduct algorithm 5 in experiments.

470

8.5. Experimental Result

Distinguished semantic association relations under different cognitive logical structures

To validate that different cognitive logical structures reflect different keyword association relations, we compare the difference of keyword association relations under different cognitive logical structures. Table 7 shows the confidence dif-

Table 7: Structure Analysis of $P S R_{\aleph}$

\begin{tabular}{|c|c|c|c|c|}
\hline \hline \multirow{3}{*}{ Data sources } & $\begin{array}{c}\text { reference structure(R) } \\
\text { conf }(C)>\operatorname{conf}(R)\end{array}$ & $\begin{array}{c}P S R_{S P I F} \\
(\%)\end{array}$ & $\begin{array}{c}P S R_{I P S F} \\
(\%)\end{array}$ & $\begin{array}{c}P S R_{I O O} \\
(\%)\end{array}$ \\
\hline \multirow{3}{*}{ DataSet1 } & $P S R_{S P I F}$ & 100 & 51.56 & 33.59 \\
\cline { 2 - 5 } & $P S R_{I P S F}$ & 48.44 & 100 & 48.44 \\
\cline { 2 - 5 } & $P S R_{I O O}$ & 58.59 & 48.44 & 100 \\
\hline & $P S R_{S P I F}$ & 100 & 52.81 & 32.45 \\
\cline { 2 - 5 } & $P S R_{I P S F}$ & 46,17 & 100 & 45.93 \\
\cline { 2 - 5 } & $P S R_{I O O}$ & 56.25 & 46.03 & 100 \\
\hline \hline
\end{tabular}

ference of association relations under different logical structures. $P S R_{S P I F}$ supports $51.56 \%$ association relations by higher confidence compared with that of $P S R_{I P S F}$ and supports $33.59 \%$ association relations compared with that of $P S R_{I O O} ; P S R_{I P S F}$ supports $48.44 \%$ association relations by higher confidence compared with that of $P S R_{S P I F}$ and supports $48.44 \%$ ones compared with that of $P S R_{I O O}$. Fig.5 show that the association relations under different cognitive logical structures have distinguished difference which decides different keyword spreading direction on association link network under different cognitive logical structures.

The shift of cognitive logical structure in decision making process Cognitive decision making process plays an important role in sentence ordering, 


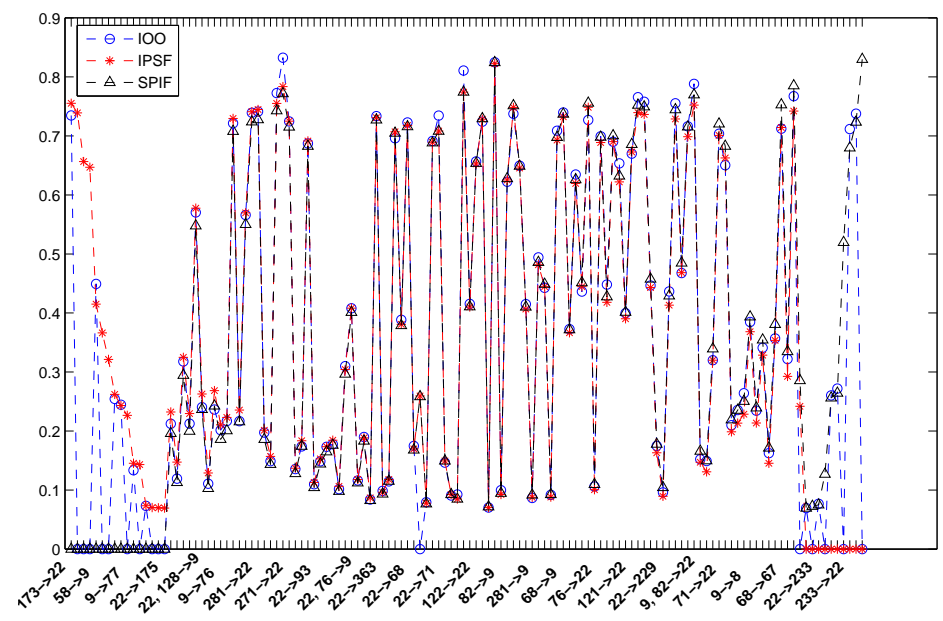

Figure 5: association relations under different logical structures

Table 8: shift of cognitive logical structure in decision making

\begin{tabular}{|c|c|c|c|c|}
\hline \hline \multirow{3}{*}{$\aleph_{(t-1)}$} & \multirow{3}{*}{$W_{(t-1)}$} & \multicolumn{3}{|c|}{$\aleph_{(t)}$} \\
\cline { 2 - 5 } & & $\aleph_{I P S F}(\%)$ & $\aleph_{S P I F}(\%)$ & $\aleph_{I O O}(\%)$ \\
\hline \multirow{3}{*}{$\aleph_{\text {IPSF }}$} & 0 & 25.39 & 12.50 & 62.11 \\
\cline { 2 - 5 } & 1 & 77.05 & 4.67 & 18.28 \\
\cline { 2 - 5 } & 2 & 22.77 & 25.32 & 51.91 \\
\cline { 2 - 5 } & $\ldots$ & $\ldots$ & $\ldots$ & $\ldots$ \\
\hline \multirow{3}{*}{$\aleph_{I O O}$} & 0 & 74.80 & 11.49 & 13.71 \\
\cline { 2 - 5 } & $\ldots$ & $\ldots$ & $\ldots$ & $\ldots$ \\
\cline { 2 - 5 } & 14 & 33.33 & 33.33 & 33.33 \\
\cline { 2 - 5 } & $\ldots$ & 58.6 & 17.20 & 42.14 \\
\cline { 2 - 5 } & 13 & 33.33 & 33.33 & 33.33 \\
\hline \hline
\end{tabular}


which is the precondition for keyword spreading process and sentence activation process. We learn the shift of cognitive logical structures by algorithm 5 from training data in dataset 1 . Table 8 and Fig 6 show the shift of cognitive logical structure in decision making learned from sentence ordering, where the probability of logical structure $\aleph_{(t)}$ is conditioned on $\aleph_{(t-1)}$ and activated keyword window $W_{(t-1)}$.

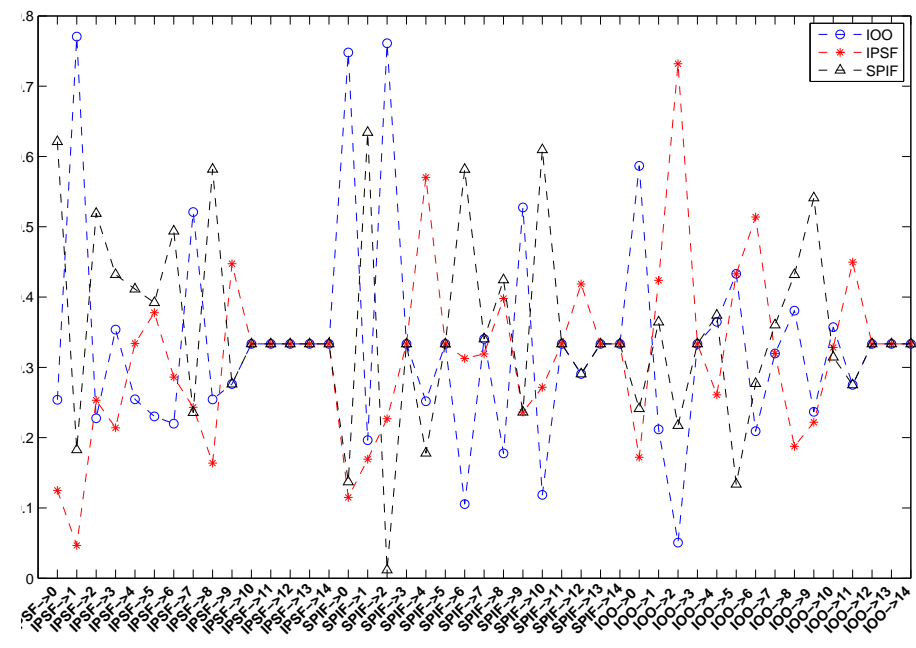

Figure 6: shift of cognitive logical structure in decision making

\section{Positive effect of cognitive logical structure on sentence order}

495 models in datasets 1-2 under combination codes 1-4. Besides, IOO-SOM has better performance than SPIF-SOM and IPSF-SOM do with which is statistically obvious since Sig. $<0.05$; SPIF-SOM dose better than IPSF-SOM.

To validate a well policy of decision making of cognitive logical has positive effect on sentence ordering model, we compare the results of our sentence ordering model with single cognitive logical structure-base sentence ordering model in section 8.3. We evaluate the results of experiment by evaluation measurement in section 8.2 and better methods will obtain higher evaluation value. The comparison results are shown in table 9 and Fig.7. 
Table 9: Comparisons of average accuracy between our model with single cognitive logical structure-based models

\begin{tabular}{|c|c|c|c|c|c|c|c|}
\hline \hline \multirow{3}{*}{ Dataset } & \multirow{2}{*}{$\begin{array}{c}\text { combination } \\
\text { code }\end{array}$} & CM-SOM & SPIF-SOM & IPSF-SOM & IOO-SOM & \multirow{2}{*}{ sig. } \\
\cline { 2 - 6 } & 0 & 52.28 & 32.17 & 14.93 & 35.17 & 0.001 \\
\hline \multirow{3}{*}{ Dataset1 } & 1 & 57.61 & 34.86 & 17.30 & 37.13 & 0.000 \\
\cline { 2 - 7 } & 2 & 58.76 & 32.17 & 14.93 & 35.17 & 0.000 \\
\cline { 2 - 7 } & 3 & 60.12 & 34.86 & 17.30 & 37.13 & 0.000 \\
\hline \multirow{3}{*}{ Dataset2 } & 1 & 47.12 & 29.71 & 24.50 & 31.72 & 0.001 \\
\cline { 2 - 6 } & 2 & 52.47 & 29.71 & 24.50 & 31.72 & 0.000 \\
\cline { 2 - 6 } & 3 & 53.81 & 25.73 & 21.44 & 34.86 & 0.000 \\
\hline \hline
\end{tabular}

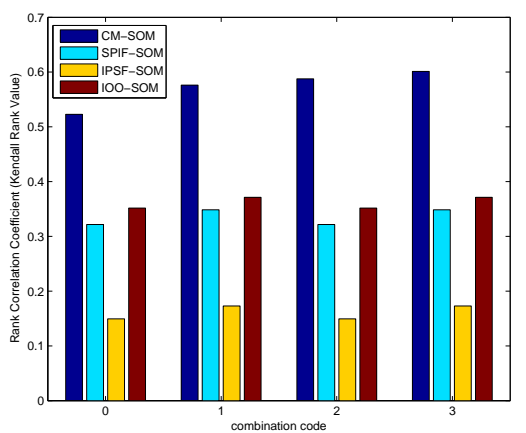

(a) Dataset 1

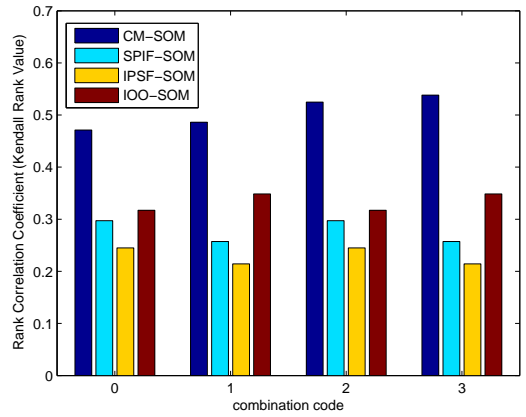

(b) Dataset 2

Figure 7: Comparisons of our model with single cognitive logical structure-based models 

mechanism

The above results validate that cognitive logical structures and their decision making policy have positive impact on sentence ordering model since the evalcodes 1-4 and run on test data of dateset 1-2. Better model will obtain higher evaluation value.

Table 10: Comparisons of average accuracy between our model with state-ofthe-art models

\begin{tabular}{|c|c|c|c|c|c|c|}
\hline \hline \multirow{3}{*}{ Dataset } & \multirow{2}{*}{$\begin{array}{c}\text { combination } \\
\text { code }\end{array}$} & CM-SOM & SBM & DBM & EBM & \multirow{2}{*}{ sig. } \\
\cline { 2 - 6 } & 0 & 52.28 & 35.74 & 46.21 & 53.43 & 0.001 \\
\hline \multirow{4}{*}{ Dataset1 } & 1 & 57.61 & 35.74 & 46.21 & 53.43 & 0.000 \\
\cline { 2 - 6 } & 2 & 57.61 & 35.74 & 46.21 & 53.43 & 0.001 \\
\cline { 2 - 6 } & 3 & 60.12 & 35.74 & 46.21 & 53.43 & 0.000 \\
\hline \multirow{3}{*}{ Dataset2 } & 0 & 47.12 & 30.14 & 39.28 & 45.43 & 0.000 \\
\cline { 2 - 6 } & 1 & 48.62 & 30.14 & 39.28 & 45.43 & 0.000 \\
\cline { 2 - 6 } & 2 & 52.47 & 30.14 & 39.28 & 45.43 & 0.001 \\
\cline { 2 - 6 } & 3 & 53.81 & 30.14 & 39.28 & 45.43 & 0.000 \\
\hline
\end{tabular}

Table 10 gives results by comparing average accuracy of our model with the state-of-the-art models including SBM, DBM and EBM. It shows that our 520 model outperforms other three models and obtains higher values of evaluation measurement. 


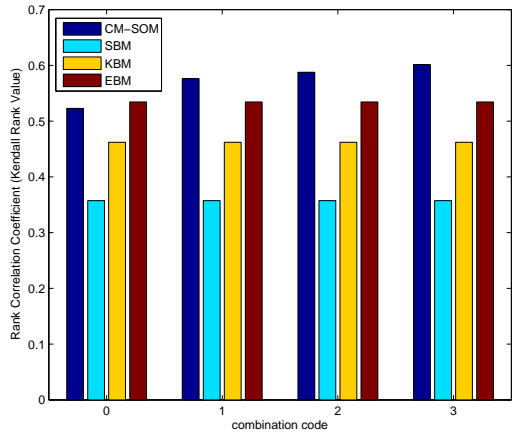

(a) Dataset 1

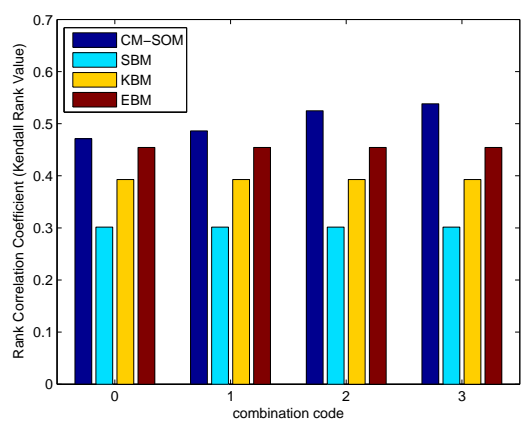

(b) Dataset 2

Figure 8: Comparisons of average accuracy between our model with state-ofthe-art models

\section{CONCLUSIONS}

Inspired by cognitive memory theory, a cognitive memory-inspired sentence ordering model is proposed to order unordered sentences. In the model, three computable cognitive logical structures are learn from texts, which include: 1) summary is prior and illustrate follows; 2) illustration is prior and summary follows and 3) illustration is one by one. Different cognitive logical structures imply different keyword association relations, by which we construct corresponding semantic markov random fields which have strong abilities in semantic representation and inference. The sentence ordering module begins with making decision of cognitive logical structure and then makes keywords spreading and sentence activation on the corresponding cognitive logical structure based markov random field.

Our sentence ordering model has solid cognitive and computational foundation. The contributions of out model are summarized as follow.

1. Inspired by human beings' common sentence cognitive logical structures, we propose machine-oriented sentence cognitive logical structures and learn them by constructing different cognitive logical trees; 
2. Inspired by cognitive memory ability in 2-degree association hypothesis, Municipal Education Commission (grant No.13ZZ064).

\section{References}

[1] R. Zhang, Sentence ordering driven by local and global coherence for summary generation, in: Proceedings of the ACL 2011 Student Session, Assostructures to infer high-order association relations in sentence ordering;

3. Simulating human beings' cognitive power in semantic representation and inference, we construct cognitive logical structure-based semantic makov random fields where semantic association relations are stored and sentence ordering is processed.

4. Simulating human beings' strong learning ability, our sentence ordering model automatically learns different cognitive structures and the structure based-association relations, structure based-semantic markov random field and decision making policy;

5. Simulating human beings' cognitive memory mechanisms that sentence ordering works on three memory modules, our sentence ordering model automatically generates a coherent sentence order by three procedures including decision making, keyword spreading and sentence activation.

So our sentence ordering model is more approaching to the human beings' cognitive memory produces and experimental results show that our model outperforms other baseline methods as well. Our model can be applied in automatic web text summarization, web short text organization and QA system.

\section{Acknowledgment}

The Research in this paper was supported by the National Science Foundation of China (grant No. 61471232), the Key Innovation Program of Shanghai

$$
\text { ciation for Computational Linguistics, 2011, pp. 6-11. }
$$
we mine low-degree association relations under different cognitive logical 
[2] M. Lapata, Probabilistic text structuring: Experiments with sentence ordering, in: Proceedings of the 41st Annual Meeting on Association for Computational Linguistics-Volume 1, Association for Computational Linguistics, 2003, pp. 545-552.

[3] R. Barzilay, L. Lee, Catching the drift: Probabilistic content models, with applications to generation and summarization, arXiv preprint cs/0405039.

[4] R. Barzilay, M. Lapata, Modeling local coherence: An entity-based approach, Computational Linguistics 34 (1) (2008) 1-34.

[5] T. Nahnsen, Domain-independent shallow sentence ordering, in: Proceedings of Human Language Technologies: The 2009 Annual Conference of the North American Chapter of the Association for Computational Linguistics, Companion Volume: Student Research Workshop and Doctoral Consortium, Association for Computational Linguistics, 2009, pp. 78-83.

[6] R. Soricut, D. Marcu, Discourse generation using utility-trained coherence models, in: Proceedings of the COLING/ACL on Main conference poster sessions, Association for Computational Linguistics, 2006, pp. 803-810.

[7] Z. Lin, H. T. Ng, M.-Y. Kan, Automatically evaluating text coherence using discourse relations, in: Proceedings of the 49th Annual Meeting of the Association for Computational Linguistics: Human Language TechnologiesVolume 1, Association for Computational Linguistics, 2011, pp. 997-1006.

[8] M. Taboada, W. C. Mann, Rhetorical structure theory: Looking back and moving ahead, Discourse studies 8 (3) (2006) 423-459.

[9] Y. Wang, A formal syntax of natural languages and the deductive grammar, Fundamenta Informaticae 90 (4) (2009) 353.

[10] E. Davoodi, On the usage of discourse relations across texts with different readability levels, in: Advances in Artificial Intelligence, Springer, 2015, pp. $347-351$. 
[11] N. Fang, X. Luo, W. Xu, Measuring textual context based on cognitive principles, International Journal of Software Science and Computational Intelligence (IJSSCI) 1 (4) (2009) 61-89.

[12] W. Liu, X. Luo, A semantic coherence based intelligent search system, in: Active Media Technology, Springer, 2013, pp. 390-400.

[13] W. Liu, X. Luo, J. Shu, How the bridging inference links unordered sentences for semantic coherence, in: Cognitive Informatics \& Cognitive Computing (ICCI* CC), 2015 IEEE 14th International Conference on, IEEE, 2015, pp. 242-249.

[14] R. Barzilay, Information fusion for multidocument summarization: paraphrasing and generation, Ph.D. thesis, Columbia University (2003).

[15] C. Fuchs, Science as a self-organizing meta-information system, Available at SSRN 504244.

[16] T. A. Hollihan, K. T. Baaske, Arguments and arguing: The products and process of human decision making, Waveland Press, 2004.

[17] D. Barbieri, D. Braga, S. Ceri, E. D. Valle, Y. Huang, V. Tresp, A. Rettinger, H. Wermser, Deductive and inductive stream reasoning for semantic social media analytics, Intelligent Systems, IEEE 25 (6) (2010) 32-41.

[18] N. J. Nersessian, Model-based reasoning in conceptual change, in: Modelbased reasoning in scientific discovery, Springer, 1999, pp. 5-22.

[19] T. A. Hollihan, K. T. Baaske, Arguments and arguing: The products and process of human decision making, Waveland Press, 2015.

[20] W. Liu, X. Luo, Z. Gong, J. Xuan, N. M. Kou, Z. Xu, Discovering the core semantics of event from social media, Future Generation Computer Systems. 
[21] X. Luo, J. Zhang, F. Ye, P. Wang, C. Cai, Power series representation model of text knowledge based on human concept learning, Systems, Man, and Cybernetics: Systems, IEEE Transactions on 44 (1) (2014) 86-102.

[22] L. Flower, J. R. Hayes, A cognitive process theory of writing, College composition and communication (1981) 365-387.

[23] R. De Beaugrande, Introduction to text linguistics.

[24] A. C. Graesser, M. Singer, T. Trabasso, Constructing inferences during narrative text comprehension., Psychological review 101 (3) (1994) 371.

[25] G. A. Miller, The magical number seven, plus or minus two: some limits on our capacity for processing information., Psychological review 63 (2) (1956) 81.

[26] D. Pavlov, H. Mannila, P. Smyth, Beyond independence: Probabilistic models for query approximation on binary transaction data, Knowledge and Data Engineering, IEEE Transactions on 15 (6) (2003) 1409-1421.

[27] F. Jelinek, Statistical methods for speech recognition, MIT press, 1997.

[28] J. N. Darroch, D. Ratcliff, Generalized iterative scaling for log-linear models, The annals of mathematical statistics (1972) 1470-1480.

[29] D. Pavlov, H. Mannila, P. Smyth, Probabilistic models for query approximation with large sparse binary data sets, in: Proceedings of the Sixteenth conference on Uncertainty in artificial intelligence, Morgan Kaufmann Publishers Inc., 2000, pp. 465-472.

[30] C. Huang, A. Darwiche, Inference in belief networks: A procedural guide, 640 International Journal of Approximate Reasoning 15 (3) (1996) 225-263.

[31] L. Kocsis, C. Szepesvári, Bandit based monte-carlo planning, in: Machine Learning: ECML 2006, Springer, 2006, pp. 282-293. 
[32] M. Kearns, Y. Mansour, A. Y. Ng, A sparse sampling algorithm for nearoptimal planning in large markov decision processes, Machine Learning 49 (2-3) (2002) 193-208.

[33] M. Toussaint, A. Storkey, Probabilistic inference for solving discrete and continuous state markov decision processes, in: Proceedings of the 23rd international conference on Machine learning, ACM, 2006, pp. 945-952.

[34] N. Vlassis, M. Toussaint, Model-free reinforcement learning as mixture learning, in: Proceedings of the 26th Annual International Conference on Machine Learning, ACM, 2009, pp. 1081-1088. 